\title{
Effective treatment for rhinopulmonary mucormycosis in a boy with leukaemia
}

\author{
O. B. EDEN AND J. SANTOS
}

Children's Hospital at Stanford and Department of Paediatrics, Stanford University Medical Centre

SUMMARY Lymphoblastic leukaemia in a 3-year-old child was complicated by rhinopulmonary mucormycosis. This was successfully treated by surgical resection of the nasal mass, pulmonary lobectomy, combined with amphotericin.

Effective treatment of childhood lymphoblastic leukaemia has been associated with an increased incidence of atypical infections (Hughes, 1971; Krick and Remington, 1976), including phycomycosis (Harris, 1955; Mirsky and Cuttner, 1972; Myer et al., 1972). Successful management of rhinopulmonary mucormycosis in childhood malignancy has not been reported.

\section{Case history}

In July 1971 , a $3 \frac{1}{2}$-year-old white boy with a leucocyte count of $7 \cdot 7 \times 10^{9} / 1$ was diagnosed as having acute lymphoblastic leukaemia (ALL). Chemotherapy was begun with daily prednisolone $\left(40 \mathrm{mg} / \mathrm{m}^{2}\right)$ and weekly vincristine $\left(1.5 \mathrm{mg} / \mathrm{m}^{2}\right)$. Once in remission at 4 weeks, central nervous system prophylaxis was given (2000 rads to the craniospinal axis over 13 days with weekly injections $\times 2$ of intrathecal methotrexate $0.5 \mathrm{mg} / \mathrm{kg}$ ). Maintenance was with daily 6mercaptopurine $\left(50 \mathrm{mg} / \mathrm{m}^{2}\right)$, weekly oral methotrexate $\left(20 \mathrm{mg} / \mathrm{m}^{2}\right)$, and cyclophosphamide $(200$ $\mathrm{mg} / \mathrm{m}^{2}$ ), with vincristine and prednisolone pulses every 3 months.

In May 1974 the child developed a right hemiparesis and a left 3 rd and right 6 th and 7 th cranial nerve palsies. Fundoscopy was normal and there was no meningismus. Investigations identified a $2-3 \mathrm{~cm}$ mass to the left of the midline at midbrain level, deviating the 3rd ventricle to the right. Lumbar thecal pressure was normal with CSF glucose $65 \mathrm{mg} /$ $100 \mathrm{ml}(3.6 \mathrm{mmol} / \mathrm{l})$ and protein $38 \mathrm{mg} / 100 \mathrm{ml}(0.38$ $\mathrm{g} / \mathrm{l}$ ), with nocells. Abnormal vascularity was confirmed on carotid arteriography. Biopsy was deferred because of the lesion site but a trial of irradiation to a total dose of 4000 rads (2500 rads to whole brain and 1500 rads to midbrain) over 18 days produced slight improvement in limb power only. This was considered to suggest a new tumour, not a leukaemic or encephalopathic lesion. Computerised axial tomography (CAT) showed an increase in tumour size.

Leukaemic therapy was stopped and oral 1-(2chloroethyl)-3 cyclohexyl-1 nitroso-urea (CCNU) began $\left(100 \mathrm{mg} / \mathrm{m}^{2}\right)$ orally every 6 weeks. Between the first two dosages the tumour enlarged by a further $20 \%$ with neovascularity, but the child remained clinically well at school. Neither bone marrow nor CSF showed evidence of leukaemic relapse until April 1976 (22 months after stopping antileukaemic treatment) when he developed a left-sided bronchopneumonia coincident with haematological relapse. Leukaemic therapy was restarted and CCNU stopped. Remission was again achieved after one month. CAT scan showed calcification without further enlargement of the midbrain lesion. One month later ( 2 years after the development of hemiparesis) he had fever and mucopurulent nasal discharge, with bilateral opacified ethmoid and maxillary sinuses, left lower lobe pulmonary infiltrates, and the left hemidiaphragm was raised. Staphylococcus aureus was initially cultured from the nose but the condition did not improve with treatment on methicillin. Nasal debridement showed a large necrotic polypoid mass containing irregular broad fungal hyphae, mostly nonseptated, suggestive of mucormycosis (Fig. 1). Cultures were negative. The mass had occupied the nares, right maxillary antrum, and ethmoid sinus, causing septal perforation. After a test dose (1 mg), amphotericin in a dose of 0.25 $\mathrm{mg} / \mathrm{kg}$ per day was begun and increased by 0.25 $\mathrm{mg} / \mathrm{kg}$ per day to a maximum dose of $1 \mathrm{mg} / \mathrm{kg}$ per day. Each dose was given in $250 \mathrm{ml} 5 \%$ dextrose with 250 units heparin and $25 \mathrm{mg}$ hydrocortisone over 6 hours. Periodically, raised blood urea, nitrogen, and creatinine levels necessitated dosage being interrupted for a maximum of 2 days. Altogether $1212 \mathrm{mg}$ was administered over 7 weeks. The nose progressively improved but despite intensive physiotherapy the lung infiltration worsened (Fig. 2). Needle aspiration, bronchoscopy, and left lower lobectomy followed. On each occasion necrotic material containing occasional branching nonseptated hyphae was obtained. All fungal cultures however were negative. Postoperative recovery was complicated by wound haematomata but no infection. No further amphotericin was given. 


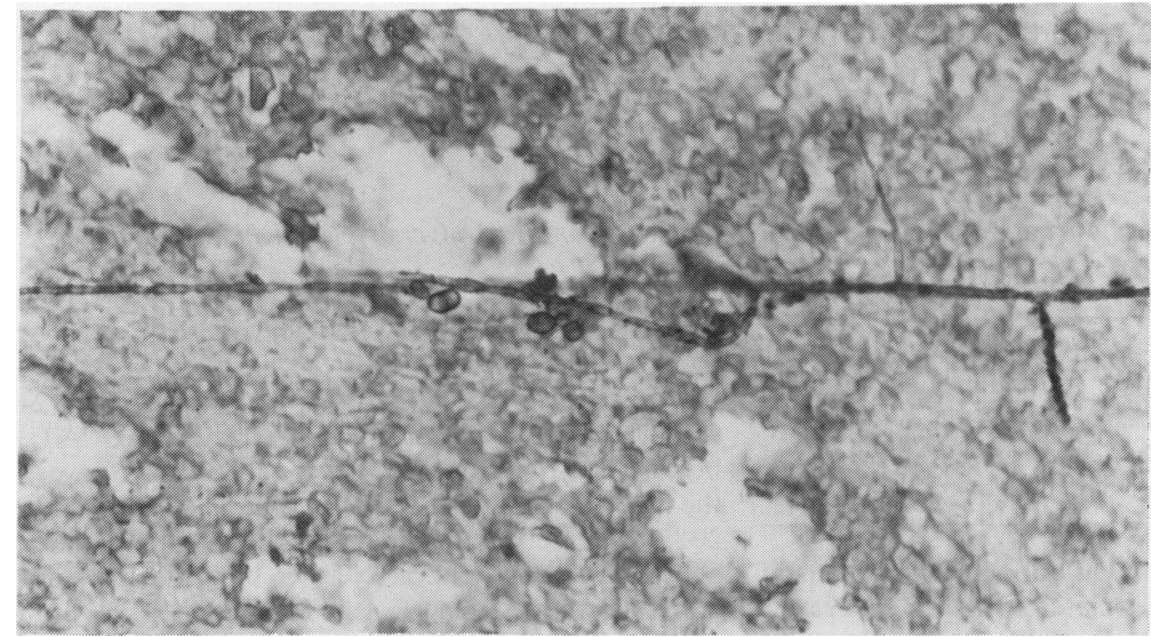

Fig. 1 Fungal hyphae identified in debris from the nares.

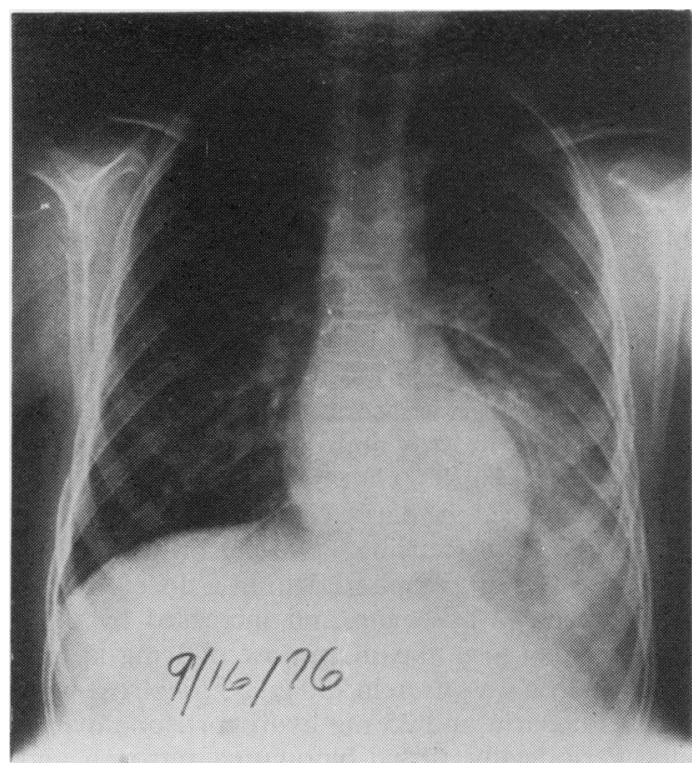

Fig. 2 Left lower lobe pneumonic consolidation.

To date ( 2 years 7 months after diagnosis of rhinopulmonary mucormycosis) the child remains well on antileukaemic chemotherapy in complete remission, but he has a persistent thalamic tumour showing contrast enhancement but no increase in size. He has no rhinorrhoea or evidence of active pulmonary disease despite a persistently paralysed left hemidiaphragm and a right hemiparesis. He attends school regularly and plays football.

\section{Discussion}

Nearly 3 years after the diagnosis of leukaemia this boy developed an intracerebral lesion and after 2 further years rhinopulmonary mucormycosis. That these two occurrences were related had to be considered. He has shown no CNS symptom progression while investigations gave no evidence of meningeal disease but showed neovascularity with late calcification. Without histology the exact nature of the mass is uncertain. Retrospectively, we have to consider rhinocerebral mucormycosis which can occur in children (Harris, 1955; McBride et al., 1960; Baker, 1962). The usual features of systemic infection of ophthalmoplegia, uncontrolled diabetes insipidus, and meningoencephalitis were all absent (Harris, 1955; McBride et al., 1960). Rapidly fatal isolated cerebral mucormycosis has been reported (Myer et al., 1972). Our patient had the lesion for at least 2 years without antifungal therapy but with continuing chemotherapy. Mucormycosis is a very aggressive infection and if untreated it will progress to death within a matter of weeks (Harris, 1955; McBride et al., 1960). Mucor hyphae were seen in nasal and lung tissue. The incidence of mycotic infections in leukaemic patients has progressively risen and now up to $30 \%$ of significant infections are of such a nature (Mirsky and Cuttner, 1972; Myer et al., 1972; Krick and Remington, 1976). Aspergillus and the phycomycetes are also showing a differential increase compared with Candida (Mirsky and Cuttner, 1972; Myer et al., 1972; Krick and Remington, 1976).

Phycomycosis normally originates in the nose and 
paranasal sinuses and characteristically invades the walls and lumen of blood vessels. In leukaemic patients it usually also invades lung and sometimes becomes disseminated (Mirsky and Cuttner, 1972; Myer et al., 1972). Any lung lobe can be affected and infarction is common (Medoff and Kobayashi, 1972). The organism is difficult to recover and grow in culture. Frequently the diagnosis is made at necropsy (Krick and Remington, 1976). It is not a common human saprophyte and therefore the presence of mucor hyphae on any biopsy smear must be taken as strong evidence of infection (Myer et al., 1972; Krick and Remington, 1976). This is especially so in a febrile leukaemic patient with a persistent and/or progressive antibiotic resistant pneumonia.

Our patient is thought to be the first child with leukaemia to be successfully treated with amphotericin and surgical resection for extensive rhinopulmonary mucormycosis. We stress the need for aggressive diagnostic procedures, including debridement and resection, in conjunction with adequate amphotericin if this diagnosis is suspected.
We acknowledge the help of Dr A. Yeager and Dr T. Long in preparing this manuscript.

\section{References}

Baker, R. D. (1962). Leukopenia and therapy in acute leukemia as factors predisposing to fatal mycosis. American Journal of Clinical Pathology, 37, 358-373.

Harris, J. S. (1955). Mucormycosis. Pediatrics, 16, 857-867.

Hughes, W. T. (1971). Fatal infections in childhood leukemia. American Journal of Diseases of Children, 122, 283-287.

Krick, J. A., and Remington, J. S. (1976). Opportunist invasive fungal infections in patients with leukaemia and lymphoma. Clinics in Haematology, 5, 249-310.

McBride, R. A., Corson, J. M., and Dammin, G. J. (1960). Mucormycosis. American Journal of Medicine, 28, 832-846.

Medoff, G., and Kobayashi, G. S. (1972). Pulmonary mucormycosis. New England Journal of Medicine, 286, 86-87.

Mirsky, H.S., and Cuttner, J. (1972). Fungal infection in acute leukemia. Cancer, 30, 348-352.

Myer, R. D., Rosen, P., and Armstrong, D. (1972). Phycomycosis complicating leukemia and lymphoma. Annals of Internal Medicine, 77, 871-879.

Correspondence to $\mathrm{Dr}$ O. B. Eden, Department of Child Life and Health, Edinburgh University, Edinburgh, Scotland.

\title{
Neonatal aspergillosis accompanying fulminant necrotising enterocolitis
}

\author{
HENRY H. MANGURTEN AND BALBINO FERNANDEZ
}

Departments of Paediatrics (Section of Neonatology) and Pathology, Lutheran General Hospital, Illinois

SUMMARY A preterm infant developed fulminant necrotising enterocolitis, subsequently resulting in death. Necropsy examination showed diffuse aspergillosis. Reports are reviewed, and possible mechanisms resulting in Aspergillus infection in this infant are discussed.

Disseminated aspergillosis in all age groups is almost always associated with an underlying chronic illness or other predisposing factors-such as prolonged administration of antibiotics or corticosteroids (Zimmerman, 1955; Luke et al., 1963; Riley, 1972). The Aspergillus is a fungus which rarely causes disease in humans. In children, particularly during the neonatal period, aspergillosis is exceedingly rare (Allan and Andersen, 1960; Luke et al., 1963). We recently unexpectedly discovered diffuse aspergillosis in a preterm infant dying from fulminant necrotising enterocolitis (NEC). We can find only five reports (see Table) of neonatal aspergillosis (Zimmerman, 1955; Akkoyunlu and Yucel, 1957;
Allan and Andersen, 1960; Matturri and Fasolis, 1962; Luke et al., 1963). We can find no previous report of this organism in association with NEC. Accordingly we describe our findings.

\section{Case report}

A 950-g boy was born by caesarean section to a 25-year-old primigravid mother after 31 weeks' gestation. Membranes ruptured spontaneously 32 days before delivery. The mother was observed in the hospital for 4 days and discharged home to rest in bed. She was readmitted 2 weeks before delivery because of peptic ulcer disease. She was treated with diazepam, diphenoxylate-hydrochloride with atropine sulphate, trimethobenzamide hydrochloride, acetaminophen, chlordiazepoxide hydrochloride with clidinium bromide, and various antacids. In addition she was given vitamins, secobarbital sodium, diphenhydramine hydrochloride, and iron dextran injection. A cholecystogram was normal. A lecithin/sphinogomyelin (L/S) ratio on amniotic 